\title{
Investigation of Effect of Low-Level Light Therapy by Continuous Wave and Pulse Wave Through Dry Skull by Using Mathematical Modeling
}

\author{
Nutchaya Thanuthep* \\ Biological Engineering Program, \\ King Mongkut's University of Technology, \\ Thonburi, Thailand
}

\author{
Anak Khantachawana \\ Mechanical Engineering, \\ King Mongkut's University of Technology, \\ Thonburi, Thailand
}

\author{
Kamolchanok Ngamkham \\ Biological Engineering Program, \\ King Mongkut's University of Technology, \\ Thonburi, Thailand
}

\begin{abstract}
Both Continuous Wave (CW) and Pulse Wave (PW) are used in Low-level Light Therapy (LLLT) treatment of skin diseases, wound healing, and hair transplantation. At present, LLLT is being used in Alzheimer's disease treatment. It was found that LLLT can prevent nerve cell death in an in-vitro test. However, LLLT for Alzheimer's disease requires high energy of light so that the light can pass through the skull and be sufficient to treat Alzheimer's disease. This study focuses on designing uniform light illumination on the skull, both CW and PW, at a wavelength of $810 \mathrm{~nm}$ using the local search algorithm without harming the skin to make the light pass through the skull.Moreover, this study measured the energy density of light when the light through the skull from the simulation. The results found that PW irradiation was able to produce more energy density than CW irradiation, and the energy density that passes the skull is enough to treat Alzheimer's disease without harming the skin. Also, the PW treatment duration was shorter than $\mathrm{CW}$, where the coefficient of variation of energy density on the skull surface of PW and CW were 0.037 and 0.036 , respectively.
\end{abstract}

Keywords: Alzheimer's disease, continuous wave irradiation, LLLT, PW irradiation, uniform illumination

Received: 10 November 2020; Accepted: 7 January 2020; Published: 25 March 2021

\section{INTRODUCTION}

Dementia is a syndrome with many causes; for example, Parkinson's disease, Vascular dementia, and the most common cause of dementia is Alzheimer's Disease (AD). In 2018, it was found that 5.7 million Americans suffer from AD [1]. This number was included an estimated 5.5 million people aged 65 and older. Furthermore, it is estimated that in 2025, people ages 65 and older will have Alzheimer's for 7.1 million people [1]. AD is caused by the accumulation of the amyloid- $\beta(\mathrm{A} \beta)$ pep- tide called amyloid plaques in the extracellular brain and the formation of neurofibrillary tangles inside neurons that interrupt the work of the brain cell, causing brain cells to die [2].

For this reason, some parts of the brains of Alzheimer's patients are slowly destroyed, starting from the hippocampus part and cerebral cortex, respectively, causing amnesia. Moreover, the ability to live is reduced. For the late stage of Alzheimer's patients, their surrounding response is diminished, and they eventually die. Cur-

\footnotetext{
${ }^{*}$ Correspondence concerning this article should be addressed to Nutchaya Thanuthep, Biological Engineering Program, King Mongkut's University of Technology, Thonburi, Thailand. E-mail: nutchayathanuthep@gmail.com

(C) 2021 The Author(s). Published by KKG Publications. This is an Open Access article distributed under a Creative Commons AttributionNonCommercial-NoDerivatives 4.0 International License.
} 
rently, there are no drugs that can completely cure AD. The drugs can only slow down the symptom. Besides, they have side effects: loss of appetite, nausea, vomiting, and diarrhea [3]. Because the efficiency of the current treatment is not high as it should be, the researchers devised a way to reduce the side effects. Recently, LLLT or photobiomodulation with near-infrared light (600-1000 $\mathrm{nm}$ ) has been used to grow hairs [4] and heal wounds [5].

Furthermore, light can reduce pain inflammatory and prevent cell death. Nowadays, LLLT has been used to treat Alzheimer's disease in in-vitro tests [6], which causes higher production of ATP in the mitochondria. As a result, the cells become more robust and can eliminate amyloid- $\beta$ (A $\beta$ ) by themself [7]. Previous research [8] found that light with a wavelength of $810 \mathrm{~nm}$ has the best penetrating capacity of human tissue. Moreover, it was found that $810 \mathrm{~nm}$ wavelength gave the best therapeutic effect on Alzheimer's disease at the cellular level [9, 10]. However, the light passes skin, skull, and brain, the energy of light is weakened by adsorption and scattering, but the light of these results is irradiated directly into the cells $[11,12]$. The light did not penetrate the skin, skull, and brain, important parameters for AD treatment. In 2011, PW irradiation was compared to continuous wave irradiation $(\mathrm{CW})$ at $810 \mathrm{~nm}$ wavelength using light irradiation to brain injury mice [13]. LLLT at $810 \mathrm{~nm}$ laser PW was found to be the most effective for improving traumatic brain injury in mice compared with continuous-wave [13]. Therefore, this research has focused on the simulation of the irradiation of LED light in BCW and PW on the skull surface and brain surface at different distances. Moreover, a low-level light panel is designed using the MATLAB program to investigate the effect of LLLT with continuous wave and PW on penetrating ability into the skull.

\section{EXPERIMENTAL}

\section{A. Curved Skull Modeling}

LLLT has been used to treat various brain diseases $[14,15]$. For AD treatment, light is necessary to pass the skull and reach brain tissue. Therefore, the thinnest part of the skull is a good choice in LLLT. The skull is divided into six main parts: frontal bone, parietal bone, sphenoid bone, temporal bone, occipital bone, and ethmoid bone, each with different thicknesses and curvature. The characteristics of the skull change according to gender, age, and race [16]. The temporal skull was considered a priority because it is the thinnest part of the skull, with a thickness of $4 \mathrm{~mm}$ [17]. Since the human skull is not uniform, it is impossible to find the cranial curvature equation. Therefore, this project used a CT scan-3D model of the human skull from a free source [18] that causes the spherical surface to overlap with the temporal skull by using Autodesk Inventor Professional 2021 program. Along with determining the boundary of the light projection on the skull in Fig. 1.

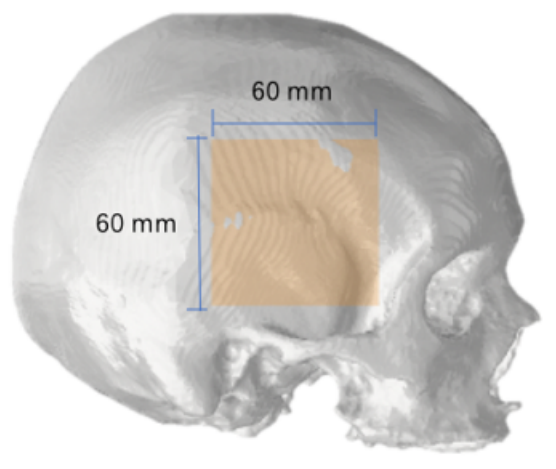

Fig. 1. The boundary of light projection on the skull

\section{B. Algorithm Design}

$810 \mathrm{~nm}$ LED with $0.16 \mathrm{~W} / \mathrm{sr}$ is used in the experiment in both $\mathrm{CW}$ and $\mathrm{PW}$, but a single LED cannot generate sufficient power for desired irradiation. Multiple LEDs are needed to increase the irradiation power, while LLLT with high energy may cause thermal damage to skin or organs. In this experiment, $10 \mathrm{~J} / \mathrm{cm}^{2}$ is the maximum energy density that skin can take without damage for CW [19] and 22.6 J/cm $\mathrm{cm}^{2}$ for PW [20]. The position of the LED affects the energy density on the skull surface. Therefore, designing and optimizing the placement of LEDs to create uniform light and high energy density are very important in the treatment of $\mathrm{AD}$. In the experiment, the dimension of the skull is $6 \times 6 \mathrm{~cm}$. The skull is divided into 100 small grids, and the size is $0.6 \times 0.6 \mathrm{~cm}$. as shown in Fig. 2 .

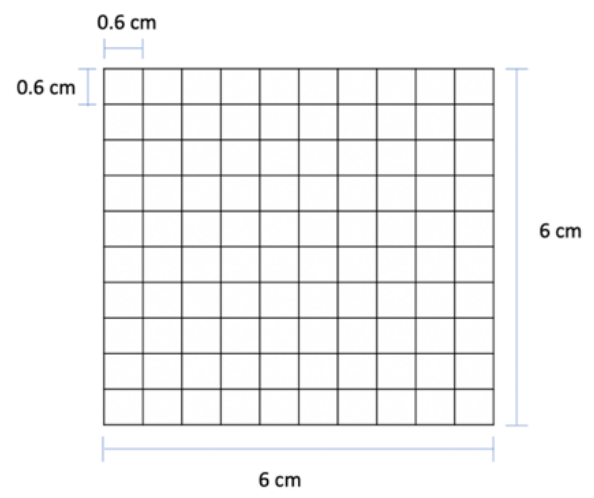

Fig. 2. Dimension and number of grids on skull

The MATLAB program is used to determine the optimum position of LED by randomizing the LED position, where it has the lowest Coefficient of Variation (CV) to ensure that the energy density on the target is equal in each area [21]. In the experiment, the different LED positions gave the different output parameters of light. Therefore, 
the LED positions are randomized with different initial conditions to find the appropriate position [22], number of LEDs (20, 30, and 40 LED), and distance between the LED panel and target $(1,2,3,4$, and $5 \mathrm{~cm})$ to be emitted to treat Alzheimer's disease. A basic flowchart of random LEDs positions is shown in Fig. 3.

The step of the flowchart is described as follows.

1. Input initial LED, skull, and air parameters as shown in Table 1.

2. Create the target plane of the skull.

3. Compare the number of LEDs and number of grids. If the number of LEDs is more than the number of grids, stop the program. If the number of LEDs is less than the number of grids, go to the next step.

4. Random LED position in grids on LED panel.

5. Determine power density and energy density of LED light on each grid at target plane and determine $\mathrm{CV}$, along with generating a random initial $\mathrm{CV}$ solution as CV star where CV star is the lowest $\mathrm{CV}$ value.
6. Set $\mathrm{k}=1$ where $\mathrm{k}$ represents LED sequence on panel (1st LED position).

7. Move the k-LED to the left, right, down, up, left-up, right-down, left-down, and right-up.

8. If the LED moves to the position where the other LED is already present, then move the LED to the next grid in line.

9. Calculate the CV value of the new LED position.

10. Compare CV value of new LED position and keep the lowest $\mathrm{CV}$ value as $\mathrm{CV}$ star.

11. Move the LED to the position where it has the lowest $\mathrm{CV}$ value.

12. Set $\mathrm{k}=\mathrm{k}+1$ (Start to move next LED sequence).

13. If LED sequence $(k)$ is less than the number of LED, then go back to step 7 .

14. If LED sequence $(k)$ more than number of LED, then print the output which show in Table 2 and end the process. 


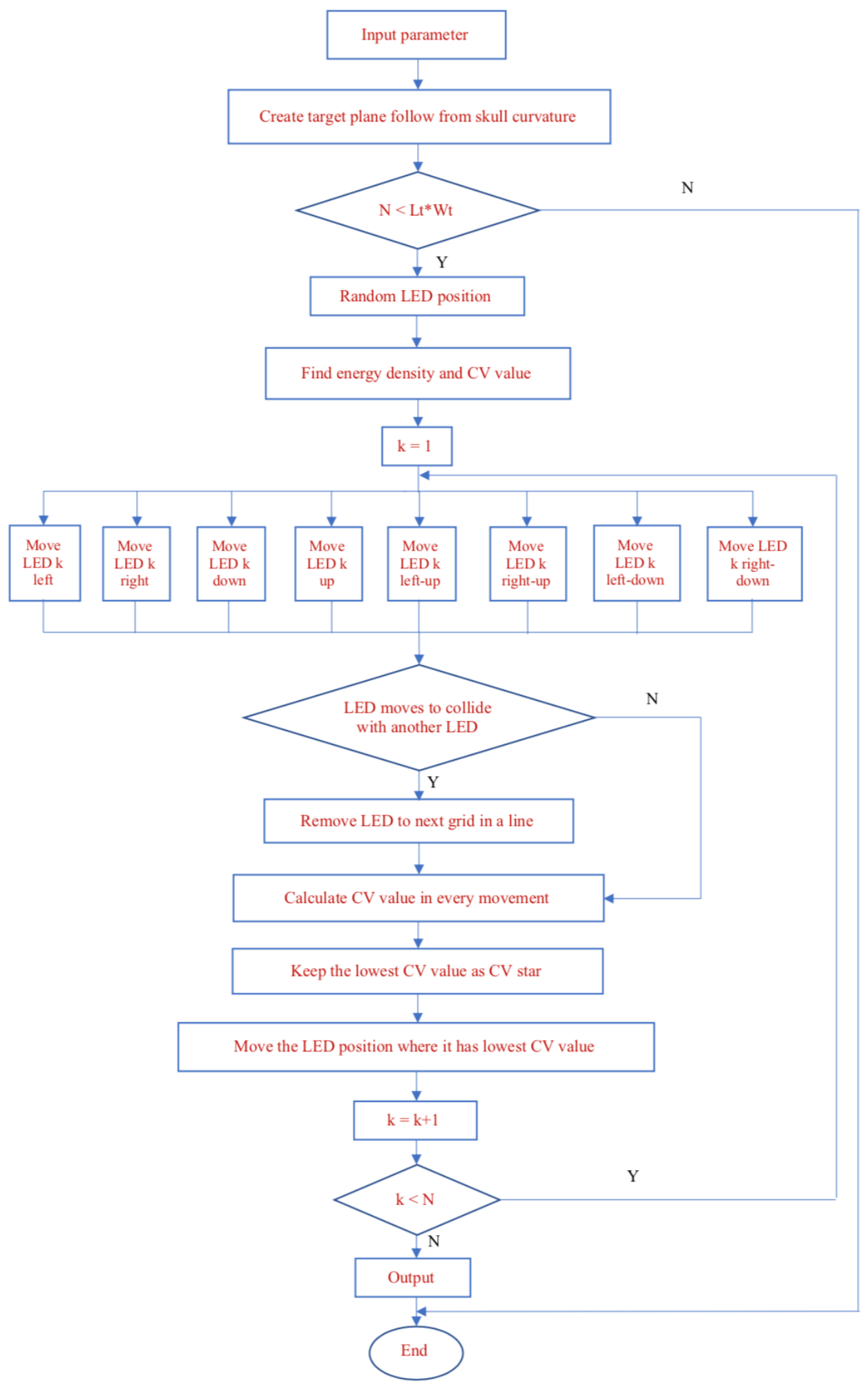

Fig. 3. Flowchart of designing LED position on panel 
TABLE 1

INPUT PARAMETERS

\begin{tabular}{|c|c|}
\hline \multicolumn{2}{|l|}{ Parameters } \\
\hline Number of LEDs $(\mathrm{N})$ & - \\
\hline The angular half-width of LED & degree \\
\hline The radiant intensity of LED & $\mathrm{W} / \mathrm{sr}$ \\
\hline Energy density at the target plane & $\mathrm{J} / \mathrm{cm}^{2}$ \\
\hline Number of grids in length $(\mathrm{Lt})$ & - \\
\hline Number of grids in width $(\mathrm{Wt})$ & - \\
\hline Grid size & $\mathrm{cm}$ \\
\hline Absorption and scattering coefficient of skin & $\mathrm{cm}^{-1}$ \\
\hline Absorption and scattering coefficient of skull & $\mathrm{cm}^{-1}$ \\
\hline Absorption and scattering coefficient of brain & $\mathrm{cm}^{-1}$ \\
\hline Distance between LED panel and target plane & $\mathrm{cm}$ \\
\hline The number of the loop cycle & - \\
\hline Skin, skull, and brain thickness & $\mathrm{cm}$ \\
\hline Frequency & $\mathrm{Hz}$ \\
\hline Duty cycle & - \\
\hline \multicolumn{2}{|l|}{$\begin{array}{c}\text { TABLE } 2 \\
\text { OUTPUT PARAMETERS }\end{array}$} \\
\hline \multicolumn{2}{|l|}{ Parameters } \\
\hline $\mathrm{CV}$ & - \\
\hline Time & $\mathrm{s}$ \\
\hline Power density at the upper surface of the skull & $\mathrm{mW} / \mathrm{cm}^{2}$ \\
\hline Power density at hippocampus & $\mathrm{mW} / \mathrm{cm}^{2}$ \\
\hline Energy density at the upper surface of the skull & $\mathrm{J} / \mathrm{cm}^{2}$ \\
\hline Energy density at hippocampus & $\mathrm{J} / \mathrm{cm}^{2}$ \\
\hline
\end{tabular}

Light travels through the skin, skull, and brain after the light hits the skull surface, and the power density is calculated. When there is an obstacle between the light emitted from the light source and the target plane, the obstacle will absorb the light and make some light unable to pass through the obstacle. Therefore, the absorption and scattering coefficient of the obstacle is considered to calculate the actual power density that passed the obstacle. The light absorption coefficient of skin, skull, and brain is $0.185,0.375,0.25 \mathrm{~cm}^{-1}$, respectively. While light scattering coefficient of skin, skull, and brain is 1.5 , $1.77,1.69 \mathrm{~cm}^{-1}$, respectively

\section{RESULTS AND DISCUSSION}

\section{A. Continuous-wave}

The LED condition results in the $\mathrm{CW}$ part consisting of CV value, the power density at the skull's outer surface, the power density at the hippocampus position in various LEDs, and the distance between LED and skull. From the results, 30 LEDs at a $4 \mathrm{~cm}$ distance between the LED and the skull showed the optimum result with the lowest $\mathrm{CV}$ value, 0.0359 . The irradiation time is 58 seconds. Power density at the outer skull surface (skin) and hippocampus is $170.8 \mathrm{~mW} / \mathrm{cm}^{2}$ and $55.3 \mathrm{~mW} / \mathrm{cm}^{2}$ Respectively, as shown in Fig. 4, where the LED position is shown in Fig. 5. The energy density at the skin surface is shown in Fig. 6. 


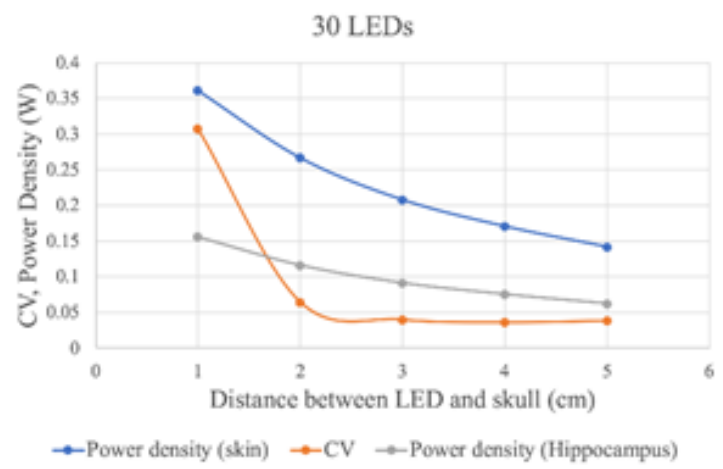

Fig. 4. CV value and power density of 30 LEDs at skin and hippocampus in various distances between LED and skull

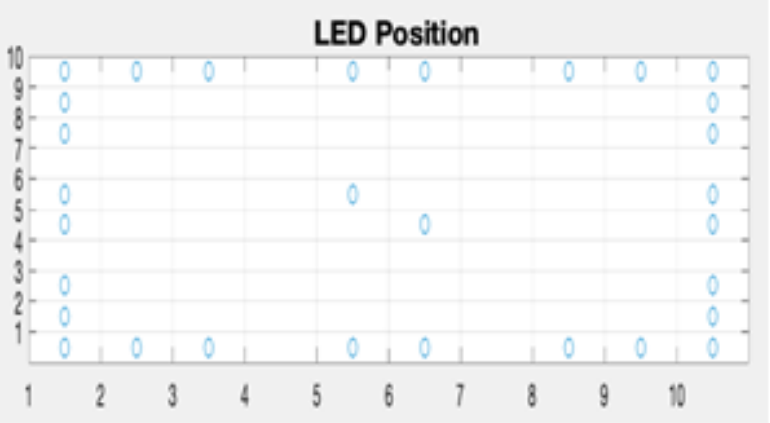

Fig. 5. The $30 \mathrm{LED}$ positions on panel in $\mathrm{CW}$ test

\section{Energy density at different distance}

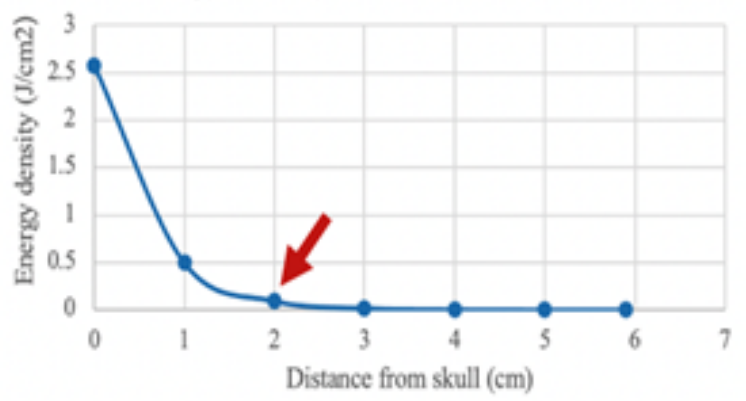

Fig. 6. Local energy density on skin surface of 30 LEDs

The light that hits the skin surface passes through the skin, skull and brain. Energy density is decreased due to absorption, scattering and thickness. It is noted that, the energy density which has ability to penetrate skin, skull and brain were calculated at the distance of $0,1,2,3,4$, $5,5.9 \mathrm{~cm}$. from the inside skull where the result is shown in Fig. 7.

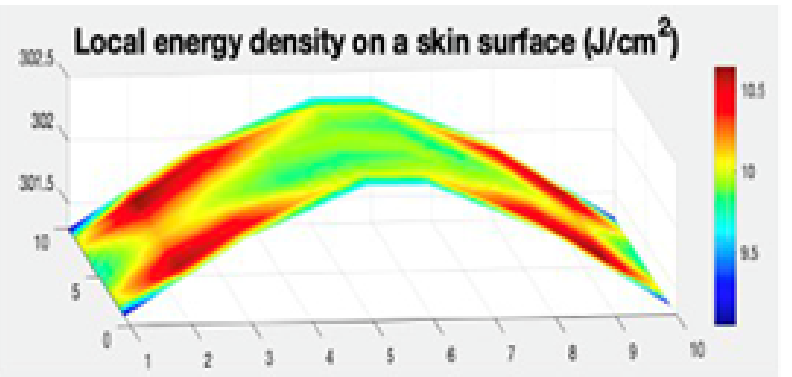

Fig. 7. Energy density of CW irradiation at different distance
However, the energy density of LLLT that affects Alzheimer's treatment is $0.03-10 \mathrm{~J} / \mathrm{cm}^{2}$ [7]. From the result, it can be said that LLLT can affect the treatment of $\mathrm{AD}$ at the brain in the range less than $0-2 \mathrm{~cm}$. from the inner skull where the energy density at $3 \mathrm{~cm}$. distance is $0.093 \mathrm{~J} / \mathrm{cm}^{2}$.

\section{B. $P W$}

In the PW part, only the results of 20 LEDs and 30 LEDs conditions were compared. In contrast, it is noted that the power density released at the outer skull surface (skin) of 40 LEDs exceeds the acceptable value of the skin. Fig. 8 shows that at 20 LEDs and the $4 \mathrm{~cm}$ distance between the LED panel and the outer skull surface is the optimal condition for PW LLLT. The lowest CV of 0.0392 can be obtained with an irradiation time of 100 seconds. Furthermore, the power density and energy density of $4 \mathrm{~cm}$. distance is not exceeded to damage the skin where power density at outer skull surface (skin) and hippocampus position is $225.7 \mathrm{~mW} / \mathrm{cm}^{2}$ and $73.1 \mathrm{~mW} / \mathrm{cm}^{2}$ Respectively, as shown in Fig. 8. The LED position is shown in Fig. 9.

\section{LEDS}

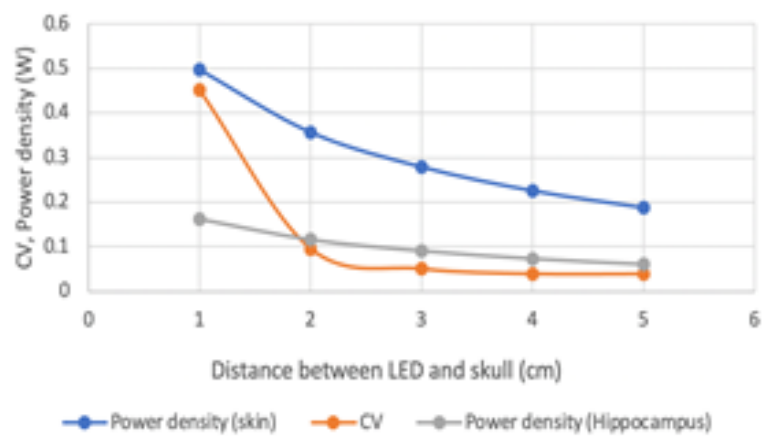

Fig. 8. CV value and power density of 20 LEDs at skin and hippocampus in various distance between LED and skull

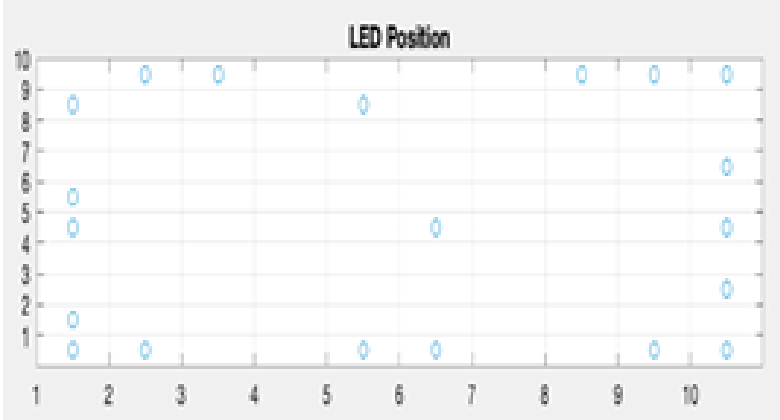

Fig. 9. The 20 LED positions on panel in PW test

Since PW does not produce heat like CW light irradiation [23], more energy without harm to the skin can be applied. The acceptable PW energy density on the skin is $22.6 \mathrm{~J} / \mathrm{cm}^{2}$, as shown in Fig. 10. The energy density of light after penetration of skin, skull and brain is shown 
in Fig. 11. From the simulation result, it was found that the energy density of PW irradiation at a distance of 0-3 $\mathrm{cm}$. from the inner skull was sufficient for the treatment of $\mathrm{AD}$ where the energy density at a distance of $3 \mathrm{~cm}$. is $0.04 \mathrm{~J} / \mathrm{cm}^{2}$.

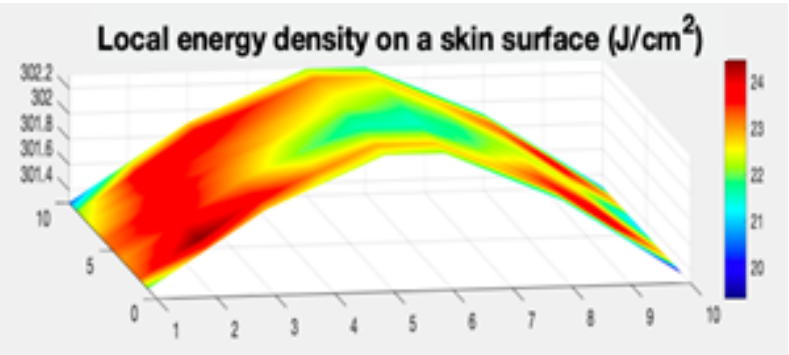

Fig. 10. Local energy density on skin surface of 20 LEDs

Energy density at different distance

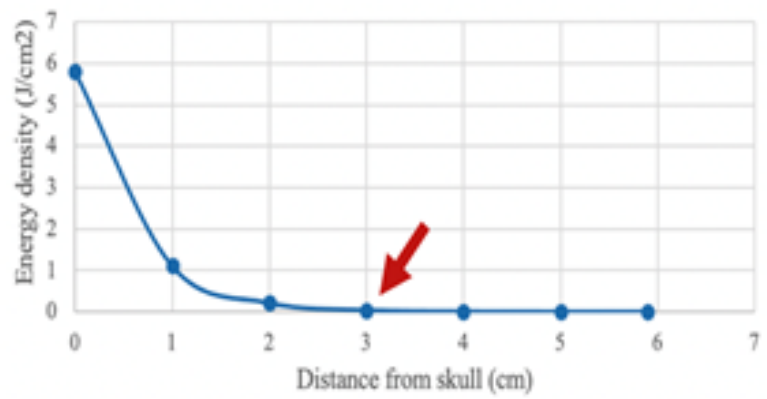

Fig. 11. Energy density of CW irradiation at different distance

\section{CONCLUSION}

In this study, the optimal LED position of continuous wave and PW conditions is designed for highly uniform light to treat Alzheimer's disease. The power density and energy density at the skin, skull, and brain were calculated by the MATLAB program to find the ability of the LED light penetration through the skull in $\mathrm{BCW}$ and $\mathrm{PW}$ to compare the effects of low-level light treatment.

Since PW irradiation does not generate heat accumulation, the skin's energy density can be obtained more than continuous wave irradiation. As a result, the simulated results of PW irradiation can penetrate the skin, skull, and brain better than continuous wave irradiation. The simulation results show that the light can reach the hippocampus, but the energy density reaching the hippocampus is insufficient to treat Alzheimer's disease compared to previous research. Therefore, LLLT may not cure the early stage of Alzheimer's disease. However, the energy density that reaches the cortex is sufficient to treat Alzheimer's disease.

\section{REFERENCES}

[1] A. Association, "2018 alzheimer's disease facts and figures," Alzheimer's \& Dementia, vol. 14, no. 3, pp.
367-429, 2018. doi: https://doi.org/10.1016/j.jalz. 2018.02.001

[2] K. L. Puig and C. K. Combs, "Expression and function of APP and its metabolites outside the central nervous system," Experimental Gerontology, vol. 48, no. 7, pp. 608-611, 2013. doi: https: //doi.org/10.1016/j.exger.2012.07.009

[3] Alzheimer's society. Effects of Alzheimer's disease drugs. [Online]. Available: https://bit.ly/3AP8Pne

[4] E. Mester, B. Szende, and P. Gärtner, "The effect of laser beams on the growth of hair in mice," Radiobiologia, Radiotherapia, vol. 9, no. 5, pp. 621-626, 1968.

[5] N. N. Houreld, "Shedding light on a new treatment for diabetic wound healing: A review on phototherapy," The Scientific World Journal, vol. 2014, pp. 1-13, 2014. doi: https://doi.org/10.1155/2014/ 398412

[6] M. H. Berman, J. P. Halper, T. W. Nichols, H. Jarrett, A. Lundy, and J. H. Huang, "Photobiomodulation with near infrared light helmet in a pilot, placebo controlled clinical trial in dementia patients testing memory and cognition," Journal of Neurology and Neuroscience, vol. 8, no. 1, pp. 1-8, 2017. doi: https://doi.org/10.21767/2171-6625.1000176

[7] S. K. Sharma, G. B. Kharkwal, M. Sajo, Y.-Y. Huang, L. De Taboada, T. McCarthy, and M. R. Hamblin, "Dose response effects of $810 \mathrm{~nm}$ laser light on mouse primary cortical neurons," Lasers in Surgery and Medicine, vol. 43, no. 8, pp. 851-859, 2011. doi: https://doi.org/10.1002/1sm.21100

[8] NeuroVault. (2016) ICBM 2009c Nonlinear Asymmetric (PD). [Online]. Available: https://neurovault.org/images/29408/

[9] Q. Wu, W. Xuan, T. Ando, T. Xu, L. Huang, Y.-Y. Huang, T. Dai, S. Dhital, S. K. Sharma, M. J. Whalen et al., "Low-level laser therapy for closed-head traumatic brain injury in mice: Effect of different wavelengths," Lasers in Surgery and Medicine, vol. 44, no. 3, pp. 218-226, 2012. doi: https://doi.org/10.1002/lsm.22003

[10] P. Avci, A. Gupta, M. Sadasivam, D. Vecchio, Z. Pam, N. Pam, and M. R. Hamblin, "Low-level Laser (light) Therapy (LLLT) in skin: Stimulating, healing, restoring," Seminars in Cutaneous Medicine and Surgery, vol. 32, no. 1, pp. 41-52, 2013.

[11] M. A. L. Bell, A. K. Ostrowski, K. Li, P. Kaanzides, and E. Boctor, "Quantifying bone thickness, light transmission, and contrast interrelationships in transcranial photoacoustic imaging," in Photons Plus 
Ultrasound: Imaging and Sensing 2015, vol. 9323. International Society for Optics and Photonics, 2015. doi: https://doi.org/10.1117/12.2078613 pp. 930-932.

[12] T. Correia, A. Gibson, and J. Hebden, "Identification of the optimal wavelengths in optical topography using photon density measurement functions," in Biomedical Applications of Light Scattering III. International Society for Optics and Photonics, San Jose, CA, 2009. doi: https://doi.org/10.1117/12. 809295

[13] T. Ando, W. Xuan, T. Xu, T. Dai, S. K. Sharma, G. B. Kharkwal, Y.-Y. Huang, Q. Wu, M. J. Whalen, S. Sato et al., "Comparison of therapeutic effects between pulsed and continuous wave 810 -nm wavelength laser irradiation for traumatic brain injury in mice," PloS one, vol. 6, no. 10, pp. 1-9, 2011. doi: https://doi.org/10.1371/journal.pone.0026212

[14] M. R. Hamblin, "Photobiomodulation for traumatic brain injury and stroke," Journal of Neuroscience Research, vol. 96, no. 4, pp. 731-743, 2018.

[15] Y.-Y. Huang, A. Gupta, D. Vecchio, V. J. B. d. Arce, S.-F. Huang, W. Xuan, and M. R. Hamblin, "Transcranial low level laser (light) therapy for traumatic brain injury," Journal of Biophotonics, vol. 5, no. 11-12, pp. 827-837, 2012. doi: https://doi.org/10.1002/jbio.201200077

[16] E. M. Lillie, J. E. Urban, S. K. Lynch, A. A. Weaver, and J. D. Stitzel, "Evaluation of skull cortical thickness changes with age and sex from computed tomography scans," Journal of Bone and Mineral Research, vol. 31, no. 2, pp. 299-307, 2016. doi: https://doi.org/10.1002/jbmr.2613

[17] H. Mahinda and O. P. Murty, "Variability in thickness of human skull bones and sternum-an autopsy experience," Journal of Forensic Medicine and Toxicology, vol. 26, no. 2, pp. 26-31, 2009.

[18] H. Newey. (2018) The anatomy of the human skull. [Online]. Available: https://bit.ly/3IYbbbq

[19] T. N. Demidova-Rice, E. V. Salomatina, A. N. Yaroslavsky, I. M. Herman, and M. R. Hamblin, "Low-level light stimulates excisional wound healing in mice," Lasers in Surgery and Medicine: The Official Journal of the American Society for Laser Medicine and Surgery, vol. 39, no. 9, pp. 706-715, 2007. doi: https://doi.org/10.1002/lsm.20549

[20] G. K. Keshri, A. Gupta, A. Yadav, S. K. Sharma, and S. B. Singh, "Photobiomodulation with pulsed and continuous wave near-infrared laser $(810 \mathrm{~nm}$, Al-Ga-As) augments dermal wound healing in immunosuppressed rats," PloS One, vol. 11, no. 11, pp. 1-21, 2016. doi: https://doi.org/10.1371/journal. pone. 0166705

[21] I. Moreno, M. Avendaño-Alejo, and R. I. Tzonchev, "Designing light-emitting diode arrays for uniform near-field irradiance," Applied Optics, vol. 45, no. 10, pp. 2265-2272, 2006. doi: https://doi.org/ 10.1364/AO.45.002265

[22] H. Yang, J. W. Bergmans, T. C. Schenk, J.-P. M. Linnartz, and R. Rietman, "Uniform illumination rendering using an array of LEDs: A signal processing perspective," IEEE Transactions on Signal Processing, vol. 57, no. 3, pp. 1044-1057, 2008. doi: https://doi.org/10.1109/TSP.2008.2009269

[23] J. T. Hashmi, Y.-Y. Huang, S. K. Sharma, D. B. Kurup, L. De Taboada, J. D. Carroll, and M. R. Hamblin, "Effect of pulsing in low-level light therapy," Lasers in Surgery and Medicine, vol. 42, no. 6, pp. 450-466, 2010. doi: https://doi.org/10.1002/lsm. 20950 